\title{
New p53 related genes in human tumors: significant downregulation in colon and lung carcinomas
}

\author{
M.E. LLEONART ${ }^{1}$, F. VIDAL ${ }^{2}$, D. GALLARDO ${ }^{2}$, M. DIAZ-FUERTES ${ }^{1}$, F. ROJO ${ }^{1}$, M. CUATRECASAS ${ }^{1}$, \\ L. LÓPEZ-VICENTE ${ }^{1}$, H. KONDOH ${ }^{3}$, C. BLANCO ${ }^{4}$, A. CARNERO ${ }^{4}$ and S. RAMÓN Y CAJAL ${ }^{1}$ \\ ${ }^{1}$ Pathology Department, Hospital Vall d'Hebron; ${ }^{2}$ Unitat de Diagnòstic i Teràpia Molecular, \\ CTBT, 119-129 Passeig Vall d'Hebron, 08035 Barcelona, Spain; ${ }^{3}$ Department of Geriatric Medicine, \\ Graduate School of Medicine, 54 Kawara-cho, Shogoin, Kyoto 606-8507, Japan; ${ }^{4}$ CNIO (Centro Nacional \\ de Investigaciones Oncológicas), c/Melchor Fernandez Almagro 3, 28029 Madrid, Spain
}

Received February 3, 2006; Accepted April 6, 2006

\begin{abstract}
Human epithelial tumors need to accumulate multiple genetic alterations to form invasive carcinomas. These genetic alterations are related with growth factor receptors, cell signalling, the cell cycle and cell invasiveness. Importantly, cells need to avoid senescence and become immortalized for this process. Recently, five genes: RPS6KA6, HDAC4, KIAA0828, TCP1 and Tip60, which modulate p53-dependent function and avoid senescence were identified in a large-scale RNA interference screen. Twenty colon, 20 prostate and 20 lung carcinomas were studied to investigate whether these genes might be related with human tumors. RNA was extracted from both normal and tumor tissue from each patient. Real-time RT-PCR was performed using TaqMan probes corresponding to the RPS6KA6, HDAC4, KIAA0828, TCP1, Tip60 and p53 genes. In colon carcinomas, the RPS6KA6, HDAC4, KIAA0828 and Tip60 genes were downregulated in tumor tissue as compared with normal tissue ( $\mathrm{P}<0.001$ for all genes). In lung carcinomas, HDAC4, KIAA0820 and Tip60 were downregulated $(\mathrm{P}<0.01, \mathrm{P}<0.001$ and $\mathrm{P}<0.001$ respectively). Whereas no significant differences were observed in prostate carcinomas, striking downregulation of the RPS6KA6 and KIAA0828 genes was observed in colon carcinomas and KIAA0828 in a subset of lung carcinomas. mRNA expression of these genes may control p53 function as well as the rasMAPK pathway, methylation and transcriptional cellular programs. These results could unravel a novel set of regulatory suppressor genes involved in human colon and lung tumors.
\end{abstract}

Correspondence to: Dr Matilde E. LLeonart, Department of Pathology, Hospital Vall d'Hebron, 119-129 Passeig Vall d'Hebron, 08035 Barcelona, Spain

E-mail: melleona@ir.vhebron.net

Key words: RPS6KA6, HDAC4, KIAA0828, TCP1, Tip60

\section{Introduction}

Malignant transformation requires an accumulation of multiple genetic alterations related with cell signaling, the cell cycle, apoptosis and cell invasiveness. Importantly, this myriad of genetic events takes place in immortalized cells (1). The genetic pathways that control senescence and orchestrate transcription of tumor suppressor genes and cell signalling are mostly unknown. Cellular senescence is activated in normal cells in response to various types of stress including telomerase uncapping, DNA damage, oxidative stress and oncogene activity (2). Both the p53 and Rb proteins play critical roles in the induction of senescence $(3,4)$. The upstream controllers of p53 in senescence include the DNA damage response pathway mediated by the ATM/ATR and Chk1/Chk2 proteins, and through the $\mathrm{p} 14^{\mathrm{ARF}}$ protein.

In the effort to detect genes related with senescence and the p53 pathway, five genes were recently identified in a largescale RNA interference screening (5). Interestingly, the genes are related with p53 activity, methylation, histone deacetylation, histone acetyl transferase and the RAS/MAP kinase pathways. The genes whose inactivation showed accelerated growth were RPS6KA6 (S6-kinase RSK4), HDAC4, KIAA0828, TCP1 (chaperonin complex polypeptide 1) and Tip60 (HIV-1 Tat interactive protein).

RPS6KA6 is a functional protein with several characteristics of the RSK family kinases. RSKs are serine kinases that are activated by growth factors and many polypeptide hormones via the ras-dependent mitogen activated protein MAP kinase cascade composed of Raf, MEK, and ERK. RSKs are central mediators of ERK in the regulation of cellular division, survival and differentiation via phosphorylation of numerous intracellular proteins $(6,7)$. However RPS6KA6 has been proposed as an inhibitor, rather than a mediator, of growth factor signal transduction (8).

At present, the only characterization performed with RSK4 has been its demonstration in various human and fetal tissue. RSK4 is a predominantly cytosolic protein expressed in the human brain, with low expression in kidney, heart, and skeletal muscle, and undetectable expression in lung, liver, spleen and pancreas (9). 
The most well characterized function of HDACs is deacetylation of core histones, which in turn leads to nucleosome compaction to repress gene transcription (10). HDACs have also been implicated in deacetylation of non-histone targets and differential regulation of HDACs have been linked to cancer (11). HDAC4 is a class II histone deacetylase reported to intervene in cell differentiation and in certain cancers, such as leukaemia (12).

The function of the KIAA0828 gene is uncertain; however it is known to contain an AHCY domain. AdoHcyHD is a ubiquitous metabolic enzyme that catalyzes the breakdown of S-adenosyl-homocysteine, a powerful inhibitor of most transmethylation reactions, to adenosine and Lhomocysteine. Methylation is an important cellular and regulatory mechanism. Protein methylation can lead to the activation or deactivation of target proteins and DNA/RNA methylation affects transcription and translation. Under pathological conditions, such as hypoxia or tissue injury, levels of S-adenosyl-homocysteine increase significantly, leading to a substantial decrease of the methylation potential within the cell $(13,14)$.

The TCP1 subunit $2 \beta$ gene, belongs to the TCP system (also called TRiC or CTT; cytoplasmic class II chaperonin tailless complex polypeptide 1). The TCP system is one of the major chaperone systems implicated in cytoplasmic protein folding in eucaryotes. This system was initially proposed to be a specialized chaperone system for the folding of actin and tubulin (15), but further experiments suggest a broader substrate spectrum in vivo involving several proteins, such as cyclin E, whose alteration may contribute to transformation (16).

Tip60 is a subunit of a protein complex involved in histone acetylation. Acetylation of the lysine residues in the histone tails by histone acetyl transferase complexes causes the histones to lose contact with the DNA, favoring activation of transcription. Tip60 shares many properties with the tumor suppressor p53 (17), the most highly targeted gene in human tumors, especially lung and colon cancer.

Tip60 and p53 proteins are involved in the cellular response to DNA damage, are subjected to proteosomal digestion following mdm2-mediated ubiquitination and accumulate after UV irradiation. Tip60 is part of a multi-molecular complex, which also contains many other proteins including Trap mediator protein and YL1 protein. Tip60 complexes have a role in chromatin double strand break repair; the loss of human Tip60 leads to an accumulation of double strand DNA breaks and is linked to a growing number of cancer types (18).

Because colon, prostate and lung carcinomas take a long time to develop and cells need to avoid senescence to become malignant, and given the fact that p53 mutations are usually a late event (19), our objective was to study upstream controller genes of p53 that might interfere with p53 function.

In this study, we used a real-time polymerase chain reaction RT-PCR technique to examine possible alterations in mRNA expression of RPS6KA6, HDAC4, KIAA0828, TCP1, Tip60 and p53 in a total of 60 patients with colon, prostate and lung carcinoma. The results constitute the first indication of a new set of genes related with p53 and senescence in human tumors.

\section{Materials and methods}

Patients. Normal tissue and tumor tissue from 20 patients with colon carcinoma, 20 patients with prostate carcinoma and 20 patients with lung carcinoma were randomly chosen from the tumor bank at the Pathology Department of Vall d'Hebrón Hospital (Barcelona, Spain). Biopsied samples are routinely collected, quickly frozen and stored at $-80^{\circ} \mathrm{C}$ immediately after surgery. All tumors were histologically examined to confirm the diagnosis of carcinoma. All procedures of the study were approved by the Ethics Committee of the Hospital Vall d'Hebron.

Real-time quantitative RT-PCR. Total RNA was extracted from normal and tumor tissue with the RNAeasy mini kit (Qiagen, Hilden, Germany). The RNA nano Lab Chip kit (Agilent, Palo Alto, CA) was used to quantify and determine the integrity of the isolated total RNA. cDNA synthesis was performed using random primers with SuperScript ${ }^{\mathrm{TM}}$ II reverse transcriptase (Invitrogen, Carlsbad, CA) and aliquots were stored at $-20^{\circ} \mathrm{C}$.

Quantitative real-time TaqMan RT-PCR technology (Applied Biosystems, Foster City, CA) was used to determine the differential expression of the selected genes. Relative quantification analysis was performed with the ABI PRISM 7700 instrument (Applied Biosystems). Data were analyzed with sequence detection software (Applied Biosystems). The PCR cycling program consisted of denaturing at $95^{\circ} \mathrm{C}$ for $10 \mathrm{~min}$ and 50 cycles at $95^{\circ} \mathrm{C}$ for $15 \mathrm{sec}$, and annealing and elongation at $60^{\circ} \mathrm{C}$ for $1 \mathrm{~min}$.

The primers and TaqMan probes (Applied Biosystems) used to analyze the six genes were: RPS6KA6 (ref. Hs00179523), HDAC4 (ref. Hs00195814), KIAA0828 (ref. Hs00379428), TCP1 (ref. Hs00197562), Tip60 (ref. Hs00197310) and TPp53 (ref. Hs00153340).

Cyclophilin (ref. 4326316E), an endogenous control, was used to normalize variations in cDNA quantities from different samples. Each reaction was performed in triplicate with cDNA from normal and tumor tissue from each patient studied. A blank sample (no DNA) was included in each experiment. A new RNA extraction was randomly performed from the original tissue of some samples and reproducible quantitative real-time PCR results were obtained (data not shown).

Sequencing. cDNA of normal and tumor tissue from 9 patients was sequenced. Six pairs of primers were designed to cover the whole codifying sequence of the KIAA0828 gene. The primers used for the cDNA sequence are described in Table I. The sequencing was performed with the BigDye terminator sequencing kit in the ABI PRISM 3700 instrument (Applied Biosystems) according to the manufacturer's instructions.

Statistical analysis. Statistical comparisons were made with the Statistical Package for Social Science, version 11.5 (SPSS, Inc., Chicago, IL). The Wilcoxon test was used to compare differences between normal and tumor tissue for each gene analyzed and the Spearman test to detect associations between genes. Lastly, to study relationships between gene expression and pathological characteristics, the non-parametric Kruskal- 
Table I. KIAA0828 sequencing. ${ }^{\mathrm{a}}$

\begin{tabular}{llc}
\hline Oligo name & \multicolumn{1}{c}{ Sequence } & AT $\left({ }^{\circ} \mathrm{C}\right)$ \\
\hline KIAA0828 A Fd & AGTCTGAGCCGG TGGTTGTC & 62 \\
KIAA0828 B Rev & GACTGAGAAATGGAACGAGAC & 62 \\
KIAA0828 C Fd & GATCCAGTTTGCTGACCAGA & 60 \\
KIAA0828 D Rev & TCATGTTTGGCTGCCAGCC & 60 \\
KIAA0828 E Fd & CCTGGAAGGGAGAGTCAGAA & 62 \\
KIAA0828 F Rev & CATACGGTCCAAGTGCTCTC & 62 \\
KIAA0828 G Fd & ATGAGGTCATCCGACAAGTG & 60 \\
KIAA0828 H Rev & AATACCTGTAGTAATTAGGCTTG & 62 \\
\hline
\end{tabular}

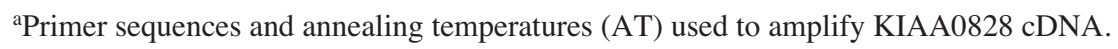
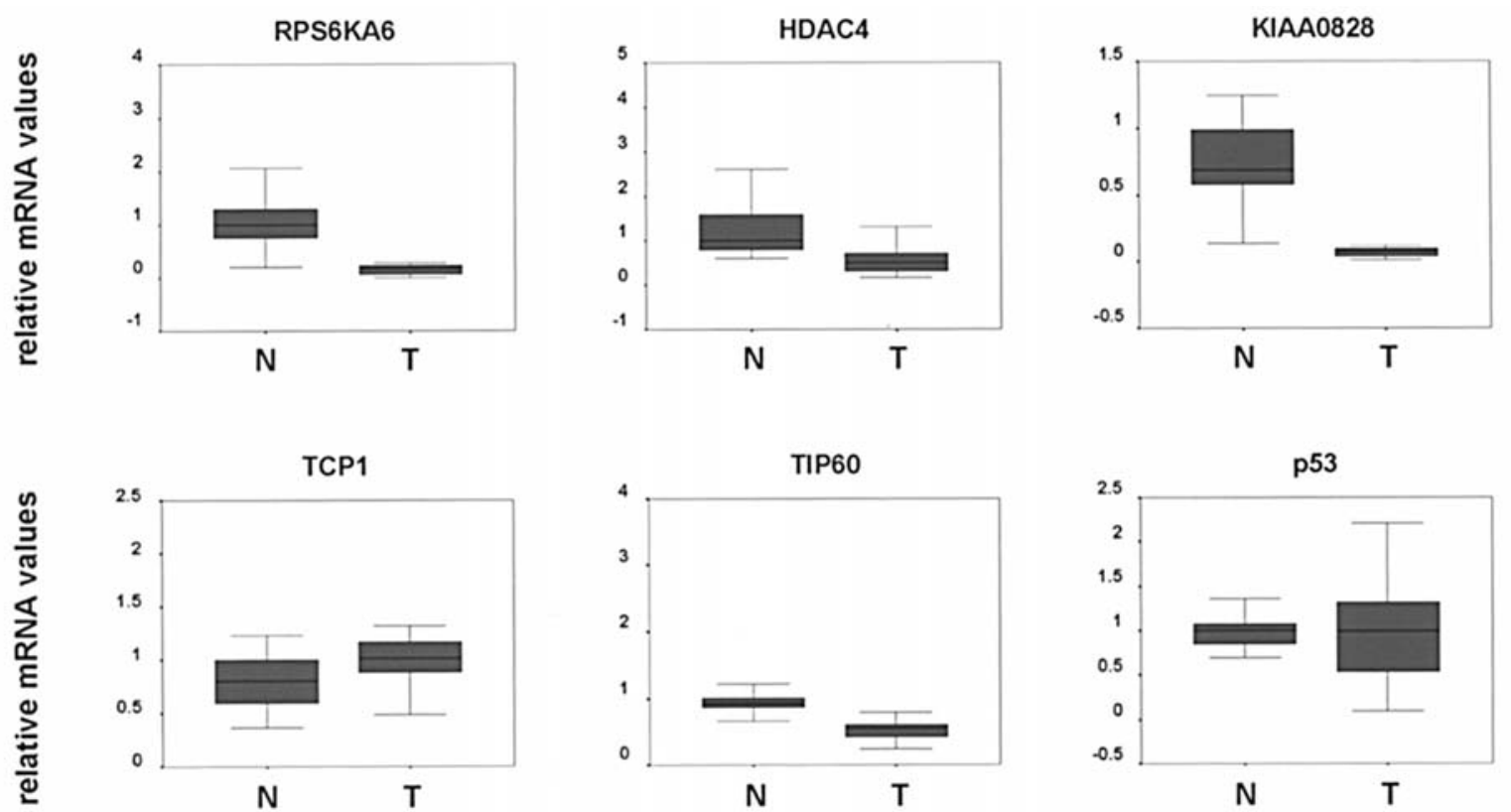

Figure 1. Colon carcinoma. Relative quantification values of mRNA levels from 20 patients with colon carcinoma. Normal (N) and tumor tissue (T) is shown for the 5 genes studied.

Wallis or Mann-Whitney U test were performed for several or two independent variables, respectively.

Only tumors with $<50 \%$ of relative mRNA compared with normal tissue were considered as down-regulated.

\section{Results}

mRNA colon cancer. The RPS6KA6, HDAC4, KIAA0828 and Tip60 genes were all found to be downregulated in colon tumor tissue (Wilcoxon test; $\mathrm{P}<0.001$ for all of them), in contrast to the TCP1 gene and p53 genes (Fig. 1). RPS6KA6 showed an $\sim 5$-fold mean reduction in mRNA in colon carcinomas as compared to normal colon tissue. There was a 10 -fold mean decrease for the KIAA0828 gene, and a 2.7- and 1.8-fold reduction, respectively, for HDAC4 and Tip60 genes relative to normal tissue. Among the 20 patients studied, RPS6KA6 was downregulated in 19, HDAC4 was down-regulated in 15,
KIAA0828 was downregulated in all 20, TCP1 was not downregulated in any case, Tip60 was downregulated in 7 and p53 was downregulated in 3 patients.

$m R N A$ prostate cancer. No differences were observed in any of the 5 genes studied in prostate cancer when comparing the relative mRNA values of each tumor sample with normal tissue from the same patient (Fig. 2). Among the 20 patients, RPS6KA6 was downregulated in 1, HDAC4 was downregulated in 3, and KIAA0828 was downregulated in 1 patient. TCP1, Tip60 and p53 were not downregulated in any patient.

mRNA lung cancer. The HDAC4, KIAA0828 and Tip60 genes were found to be downregulated in lung tumor tissue (Wilcoxon test; $\mathrm{P}<0.01, \mathrm{P}<0.001$ and $\mathrm{P}<0.001$ respectively), in contrast to the RPS6KA6, TCP1 and p53 genes (Fig. 3). HDAC4, KIAA0828 and Tip60 showed a mean reduction of 

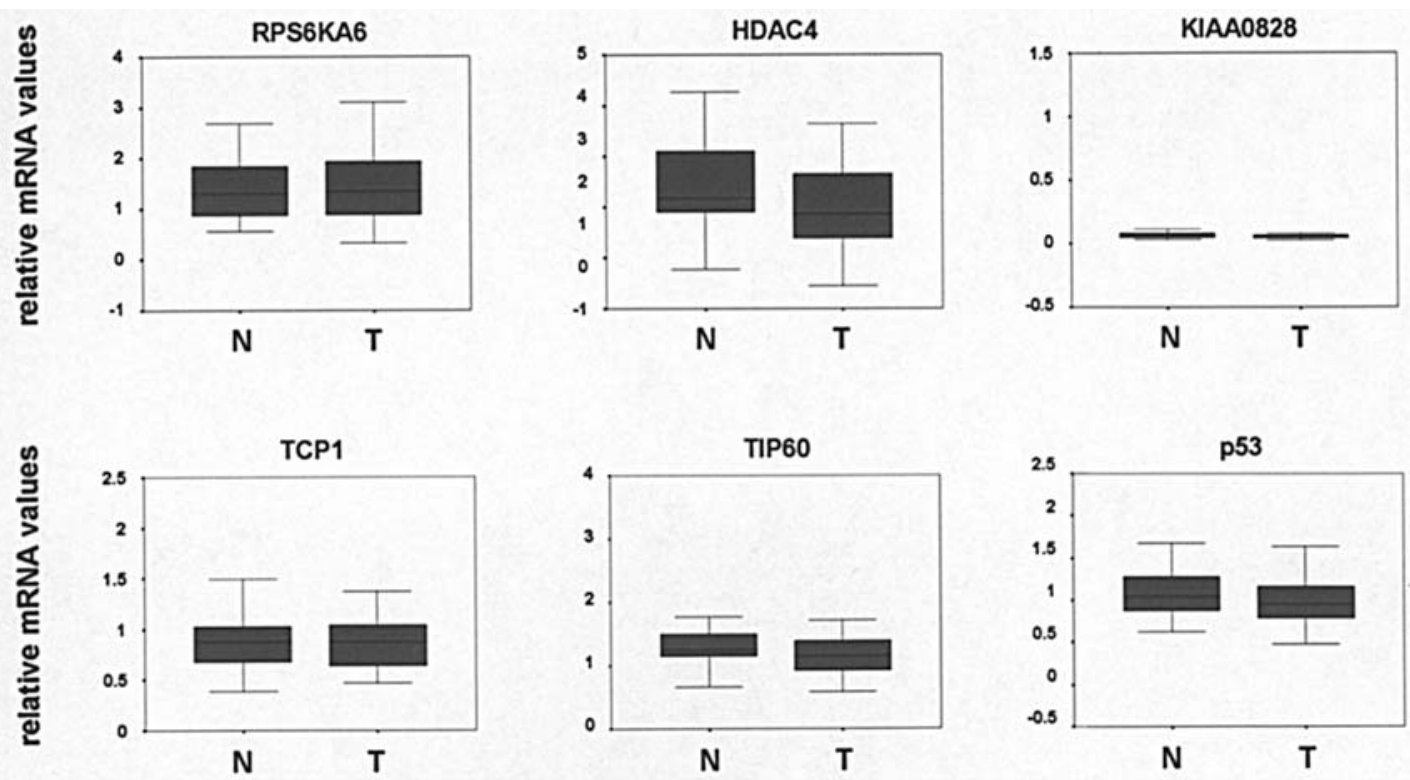

Figure 2. Prostate carcinoma. Relative quantification values of mRNA levels from 20 patients with prostate carcinoma. Normal (N) and tumor tissue (T) is shown for the 5 genes studied.
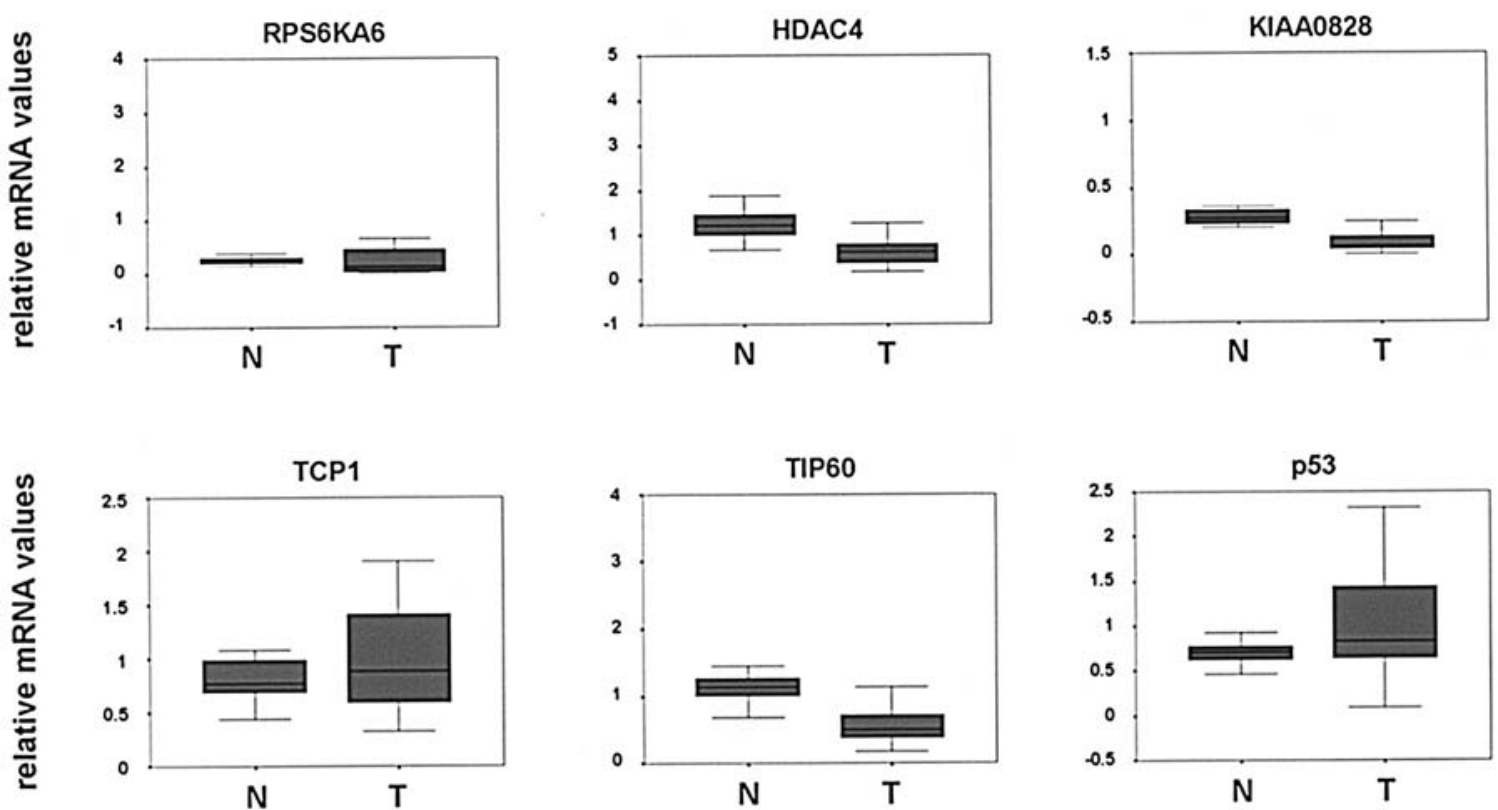

Figure 3. Lung carcinoma. Relative quantification values of mRNA levels from 20 patients with lung carcinoma. Normal (N) and tumor tissue (T) is shown for the 5 genes studied.

1.8-fold, 3.2-fold and 2-fold, respectively, as compared to normal tissue.

Among the 20 patients studied, RPS6KA6 was downregulated in 8, HDAC4 was downregulated in 10, KIAA0828 was downregulated in 15, TCP1 was not downregulated in any case, Tip60 was downregulated in 8 and p53 was downregulated in 5 patients.

KIAA0828 cDNA sequencing. Specimens from 7 patients with colon cancer and 2 patients with lung cancer were sequenced for the KIAA0828 gene. We did not observe any differences when comparing normal versus tumor tissue for each of the 9 patients analyzed (data not shown).
Pathological characteristics. No clinicopathological correlation was observed in patients with mRNA downregulation for any of the genes analyzed. The following parameters were considered: tumor size, infiltration, Dukes stage, presence of lymph node metastases, survival, TNM and PSA value in the case of prostate tumors.

\section{Discussion}

Human cancer cells exhibit a large number of genes whose overexpression or lack of expression is involved in malignant transformation. Since loss of p53 and p16 function are described as hallmarks in avoiding senescence $(3,4)$ and since 
mutated p53, frequently observed in human tumors, is detected at late stages (19), we hypothesized that other genetic alterations are related with a lack of p53 function in early stages of malignant transformation.

In a recent in vitro study, the knock-down of RPS6KA6, HDAC4, KIAA0828, TCP1, Tip60 mRNA was reported to bypass replicative senescence in IMR 90 cells and to block p53 function (5). In order to study whether these genes could be affected in human tumors, we examined alterations in their mRNA expression using real-time quantitative RT-PCR in 20 patients with colon cancer, 20 patients with prostate cancer and 20 patients with lung cancer.

RPS6KA6 has been proposed as an inhibitor, rather than a mediator of growth factor signal transduction (8) and these functional differences with respect to other members of the same family may reside in its specific N-terminal domain. Our data support the role of RPS6KA6 as an inhibitor of proliferation, since this gene was downregulated (5-fold mean mRNA decrease) in tumor tissue from 19 out of 20 patients with colon cancer. This fact suggests that a loss of RPS6KA6 function could confer an oncogenic advantage on normal colon cells, allowing them to grow and proliferate until other alterations contribute to the formation of a tumor.

The most striking finding in our study was the significant reduction in KIAA0828 mRNA levels in both colon and lung carcinomas with respect to normal tissue. Hypermethylation of $\mathrm{CpG}$ islands has been observed in many independent loci in colon cancer, but no methyltransferase gene has been directly associated with this alteration (20). Given the fact that KIAA0828 contains a domain that affects AdoHcyHD, KIAA0828 might be involved in changes in DNA methylation and thereby influence the regulation of gene expression. Interestingly, KIAA0828 mRNA was strongly downregulated in all patients with colon carcinoma (10-fold reduction) and in 15 out of 20 patients with lung carcinoma (3.2-fold reduction). These results show its potential importance given its significance in two unrelated types of cancer. Our results support the potential role of KIAA0828 as a tumor suppressor gene, as previously suggested in in vitro and experimental studies (5, Martinez-Leal et al, unpublished observations). The recent description of metabolic enzymes directly implicated in proliferation may reveal new pathways by which cancer cells grow and become immortalized (21).

After observing that KIAA0828 mRNA was strikingly decreased in colon and lung carcinoma, we proceeded to search for mutations in the cDNA sequence, No KIAA0828 gene point mutations in tumor versus normal tissue were found in the 9 patients analysed (data not shown), supporting the concept that KIAA0828 is under the control of physiological expression machinery and is not a tumor suppressor gene in the classical sense. Ongoing studies are in progress to analyze KIAA0828 mRNA expression in a wide range of tumors from benign colon adenomas to metastatic colon carcinomas to observe at which stage of tumor development the mRNA downregulation occurs.

HDAC4 was downregulated in 15 out of 20 patients with colon carcinoma $(\mathrm{P}<0.001)$ and 10 out of 20 with lung cancer $(\mathrm{P}<0.01)$. Acetylation and deacetylation in the cell is the result of a complex interaction of a number of proteins that modulate gene expression. Two out of the 5 genes previously described
HDAC4 and Tip60, have two opposite functions. Further studies are needed to determine whether HDAC4 is associated with Tip60 in human cancer. Loss of human Tip60 leads to double-strand breaks and is linked to a growing number of cancer types (18). Tip60 was found to be significantly downregulated in 7 out of 20 colon carcinomas and in 8 out of 20 lung carcinomas $(\mathrm{P}<0.001$ in both cases). Tip60 mRNA and protein downregulation was recently described in metastatic prostate cell lines when compared with normal or non-metastatic tumor cells (22). The significance obtained when comparing mRNA levels in normal versus colon and lung tumor tissue in the present study support further investigation into Tip60 as an interesting suppressor gene.

As compared to normal tissue, no statistical differences in TCP1 mRNA levels were found in colon, prostate or lung carcinomas. Although no differences in mRNA levels were documented in our study for TCP1 mRNA (Figs. 1, 2 and 3), this does not rule out an association between the TCP1 protein and human cancer as statistical differences were also absent for the p53 gene. Ongoing immunohistochemistry and Westernblotting studies are in progress to evaluate the protein levels of these genes in human tumors.

In vitro experiments have demonstrated that inactivation of each of these genes independently sufficed to increase cellular proliferation (5), therefore it is important to know whether the altered mRNA expression of the five genes studied is an early event or a consequence of alterations in other previously mutated genes.

In summary, in this report for the first time, strong downregulation of the RPS6KA6, KIAA0828 and Tip60 genes was shown in human colon and lung carcinomas. We hypothesize that these alterations are associated with the main biochemical pathways in cancer, which could drive cells to accelerated malignant transformation. Downregulation of these genes may allow cells to become immortalized and receptive to the appearance of other constitutive oncogenic alterations. These findings open new insights in cancer research and in the understanding of new senescence genes in human tumors.

\section{Acknowledgements}

We would like to thank René Bernards for his scientific support. We are grateful to T. Osteso, P. Gallego, J. Jimenez, S. Rodriguez, and E. Llonch for technical assistance and to J.A. Leal for support in the statistical analysis. The current study was supported by grants from the Ministerio de Sanidad, Ministerio de Ciencia y Tecnologia and a Long-term European Marie-Curie grant.

\section{References}

1. Hanahan D and Weinberg R: The hallmarks of cancer. Cell 100: 57-70, 2000.

2. Ben-Porath and Weinberg R: The signals and pathways activating cellular senescence. Int J Biochem Cell Biol 37: 961-976, 2005.

3. Serrano M, Lin AW, McCurrach ME, Beach D and Lowe SW: Oncogenic ras provokes premature cell senescence associated with accumulation of p53 and p16 INK4A. Cell 88: 593-602, 1997.

4. Artandi SE and Attardi LD: Pathways connecting telomeres and p53 in senescence, apoptosis, and cancer: Biochem Biophys Res Commun 331: 881-890, 2005.

5. Berns K, Hijmans EM, Mullenders J, et al: A large-scale RNAi screen in human cells identifies new components of the p53 pathway. Nature 428: 431-437, 2004. 
6. Nebreda AR and Gavin AC: Perspectives: signal transduction. Cell survival demands some Rsk. Science 286: 1309-1310, 1999.

7. Roux PP, Richards SA and Blenis J: Phosphorylation of p90 ribosomal S6 kinase (RSK) regulates extracellular signalregulated kinase docking and RSK activity. Mol Cell Biol 23: 4796-4804, 2003.

8. Myers AP, Corson LB, Rossant J and Baker JC: Characterisation of mouse RPS6KA6 as an inhibitor of fibroblast growth factor-RAS-extracellular signal-regulated kinase signalling. Mol Cell Biol 24: 4255-4266, 2004.

9. Dummler BA, Hauge C, Silber J, et al: Functional characterisation of RSK4, a new $90 \mathrm{kDa}$ ribosomal S6 kinase, reveals constitutive activation in most cell types. J Biol Chem 280: 13304-13314, 2005.

10. Yang XJ and Seto E: Collaborative spirit of histone deacetylases in regulating chromatin structure and gene expression. Curr Opin Genet Dev 13: 143-153, 2003.

11. Glaser KB, Li J, Staver MJ, Wei RQ, Albert DH and Davidsen SK: Role of class I and class II histone deacetylases in carcinoma cells using siRNA. Biochem Biophys Res Commun 310: 529-536, 2003.

12. Chauchereau A, Mathieu M, de Saintignon J, et al: HDAC4 mediates transcriptional repression by the acute promyelocytic leukaemia-associated protein PLZF. Oncogene 23: 1-8, 2004.

13. Dekker JW, Budhia S, Angel NZ, et al: Identification of an Sadenosylhomocysteine hydrolase-like transcript induced during dendritic cell differentiation. Immunogenetics 53: 993-1001, 2002.
14. Kloor D and Osswald H: S-adenosylhomocysteine hydrolase as a target for intracellular adenosine action. Trends Pharmacol Sci 25: 294-297, 2004.

15. Ritco-Vonsovici M and Willison KR: Defining the eukaryotic cytosolic chaperonin-binding sites in human tubulins. J Mol Biol 304: 81-98, 2000.

16. Won KA, Schumacher RJ, Farr GW, Horwich AL and Reed SI: Maturation of human cyclin $E$ requires the function of eukaryotic chaperonin CCT. Mol Cell Biol 18: 7584-7589, 1998.

17. Legube G, Linares LK, Tyteca S, et al: Role of the histone acetyl transferase Tip60 in the p53 pathway. J Biol Chem 43: 44825-44833, 2004

18. Ikura T, Ogryzko VV, Grigoriev M, et al: Involvement of the TIP60 histone acetylase complex in DNA repair and apoptosis. Cell 102: 463-473, 2000

19. Vousden KH and Prives C: P53 and prognosis: new insights and further complexity. Cell 120: 7-10, 2005.

20. Cindy AE, Danenberg KD, Kawakami K, Saltz LB, Danenberg PV and Laird PW: CpG island hypermethylation in human colorectal tumors is not associated with DNA methyltransferase overexpression. Cancer Res 59: 2302-2306, 1999.

21. Kondoh H, LLeonart ME, Gil J, et al: Glycolytic enzymes can modulate cellular life span. Cancer Res 65: 177-185, 2005.

22. Kim JH, Kim B and Cal L: Transcriptional regulation of a metastasis suppressor gene by Tip60 and $\beta$-catenin complexes. Nature 434: 921-926, 2005. 